Short communication:

\title{
pH-Dependence of the morphology of micelles formed by poly(2-vinylpyridine)-block-poly(ethylene oxide) copolymers in water
}

\author{
Jean-François Gohy ${ }^{1}$, Sandrine Mores ${ }^{1}$, Sunil K. Varshney ${ }^{2}$, Jian-Xin Zhang ${ }^{2}$, \\ Robert Jérôme ${ }^{1 *}$ \\ ${ }^{1}$ Centre for Education and Research on Macromolecules (CERM) - Institute of \\ Chemistry B6, University of Liège, Sart-Tilman, 4000 Liège, Belgium; \\ Fax +32 4 / 3663497; rjerome@ulg.ac.be \\ ${ }^{2}$ Polymer Source, 771 Lajoie Street, Dorval, Québec, H9P 1G7, Canada; \\ Fax +1 514 / 4229843; contact@polymersource.com
}

(Received: February 21, 2002; published: April 26, 2002)

\begin{abstract}
The morphology of micelles formed by two poly(2-vinylpyridine)-blockpoly(ethylene oxide) (P2VP-b-PEO) copolymers has been studied in phosphatebuffered water by dynamic light scattering and transmission electron microscopy. Spherical micelles are observed when the P2VP block is the minor component of the diblock copolymer. When P2VP dominates the composition, transition from spheres-to-rods-to-vesicles takes place in a narrow $\mathrm{pH}$ range that straddles the $\mathrm{pK}$ of P2VP. This transition is controlled by the degree of protonation of the P2VP blocks. At high $\mathrm{pH}$, the copolymer precipitates from the solution.
\end{abstract}

\section{Introduction}

Amphiphilic block copolymers dissolved in a solvent selective for one block usually form micelles or colloidal aggregates [1]. Water is commonly used as a selective solvent, because many valuable systems are operating in aqueous media, e.g., in the fields of coatings [2] and drug delivery [3]. Most often, micelles consist of a spherical core formed by insoluble blocks surrounded by a shell of solvated blocks. More recently, micelles with a variety of structures have been observed, including rods, lamellae, vesicles, and tubules [4-6]. Although these micelles can be formed by direct dissolution of the amphiphilic diblock copolymer in water [4,5], an organic co-solvent is often used in the dissolution step [6]. For instance, Eisenberg et al. have dissolved highly asymmetric amphiphilic diblocks in a common solvent for the two blocks (most often, $N, N$-dimethylformamide) followed by the addition of water until the major block becomes insoluble. Aggregates of various morphology that consist of a core of the insoluble block surrounded by a thin shell of the short soluble block are then formed and are called "crew-cut". They are kinetically frozen by dialysis of the organic solvent against water [6].

A more straightforward way to prepare polymeric micelles in water is to consider polymeric amphiphiles made of two blocks soluble in water, although in a limited range of $\mathrm{pH}$. Self-aggregation occurs merely upon changing the $\mathrm{pH}$, until one block 
becomes insoluble. For instance, Webber et al. reported that poly(2-vinylpyridine)block-poly(ethylene oxide) diblock copolymers (P2VP-b-PEO) formed unimers at low $\mathrm{pH}$ and micelles at $\mathrm{pH}$ higher than 4.8 [7]. This concept of $\mathrm{pH}$-dependent micellization was also illustrated by other copolymers, including poly(methacrylic acid)-blockpoly(ethylene oxide) diblocks [8]. The field of "double hydrophilic" copolymers to which these systems belong has been recently reviewed [9].

Whenever P2VP is a constitutive block of these $\mathrm{pH}$-sensitive diblocks, the exact mechanism of micelle formation might be more complex than expected because homo-P2VP is known for aggregation at least at a critical degree of ionization [10].

\section{Results and discussion}

This contribution aims at reporting on the morphology of micelles formed by two asymmetric P2VP-b-PEO diblock copolymers of different composition (see Exptl. part). Self-association of these diblocks was analyzed as a function of $\mathrm{pH}$ by dynamic light scattering (DLS). Since the two constituting blocks are water-soluble at low $\mathrm{pH}$, these diblocks form unimers in dilute acidic solution. P2VP(4.3)-b-PEO(9.0) - the numbers in parentheses being $\bar{M}_{n} \times 10^{-3}$ - shows however reversible $\mathrm{pH}$-dependent micellization, in good agreement with previously reported observations $[7,11,12]$. Indeed, micelles with a hydrodynamic radius $\left(R_{\mathrm{h}}\right)$ of $c .25 \mathrm{~nm}$ and a rather broad polydispersity index (0.2) are formed at $\mathrm{pH}>5$, the P2VP block being then essentially uncharged and thus hydrophobic. These micelles are stable at $\mathrm{pH}>7$, as reported elsewhere [11]. In sharp contrast, P2VP(26.4)-b-PEO(5.9) precipitates from the solution at $\mathrm{pH} \geq 5.5$, because the minor PEO block is too short to stabilize micelles with a core formed by the major hydrophobic P2VP block. However, the careful control of $\mathrm{pH}$ in the $\mathrm{pH} 4.8$ - 5.5 range allows stable micelles or aggregates to be observed. $R_{\mathrm{h}}$ of these objects and their polydispersity index are listed in Tab. 1.

Tab. 1. Dynamic light scattering data for the P2VP(26.4)-b-PEO(5.9) copolymer at different $\mathrm{pH}$ 's

\begin{tabular}{ccc}
\hline $\mathrm{pH}$ & $R_{\mathrm{h}} / \mathrm{nm}$ & Polydispersity index \\
\hline 4.90 & 64 & 0.10 \\
5.03 & 95 & 0.22 \\
5.11 & 103 & 0.25 \\
5.19 & 117 & 0.22 \\
5.27 & 135 & 0.35 \\
5.38 & 141 & 0.40
\end{tabular}

Except for the measurement at $\mathrm{pH} 4.9$, the polydispersity index is high and the characteristic size is too large to fit the structure of classical star-like micelles. Clearly large micellar aggregates are formed. In parallel, the P2VP(26.4)-b-PEO(5.9) diblock only forms stable aggregates when a phosphate buffer is used to set the $\mathrm{pH}$. Whenever an acidified P2VP(26.4)-b-PEO(5.9) solution is titrated by $\mathrm{NaOH}$, these aggregates are not observed, which indicates that the phosphate salt must contribute to the stabilization.

The morphology of the micelles and aggregates has been analyzed by transmission electron microscopy (TEM). Fig. 1 shows that the P2VP(4.3)-b-PEO(9.0) sample 
forms polydisperse spherical micelles at $\mathrm{pH}>5$, with an average size which is in good agreement with DLS data.

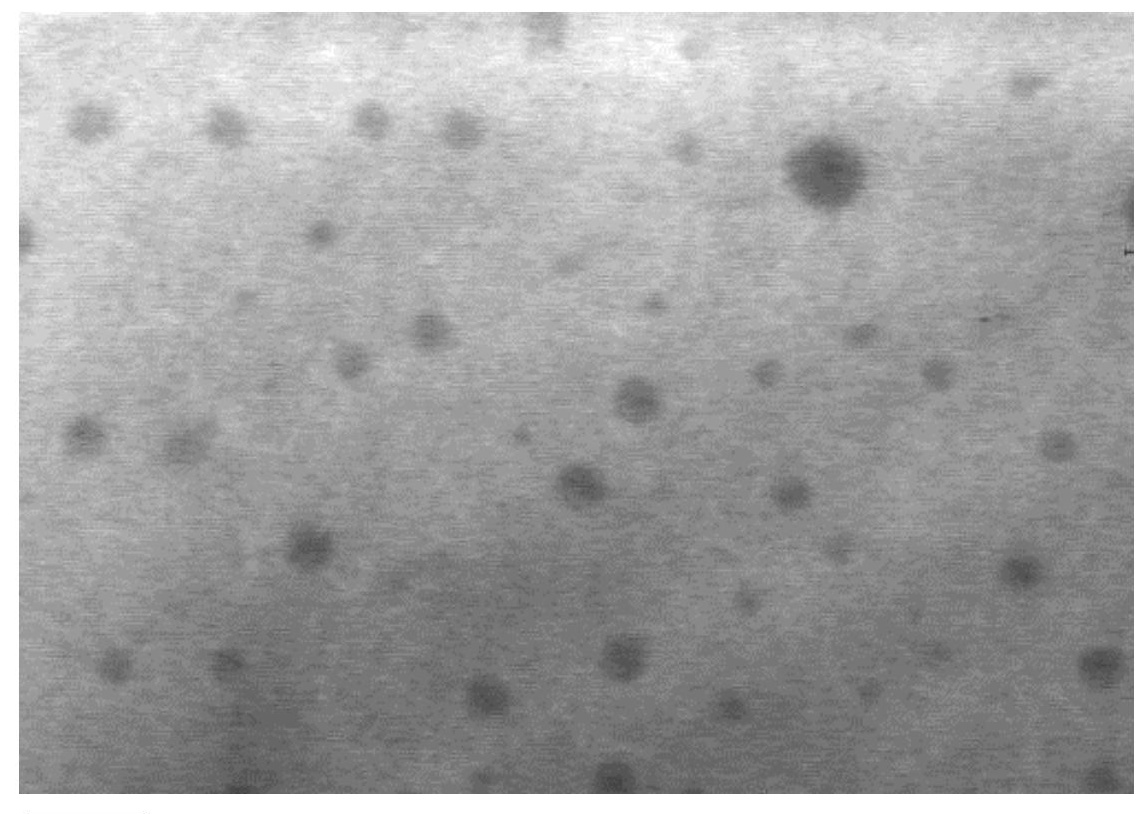

$100 \mathrm{~nm}$

Fig. 1. TEM picture of the micelles formed by the P2VP(4.3)-b-PEO(9.0) copolymer in water at $\mathrm{pH} 6.50$

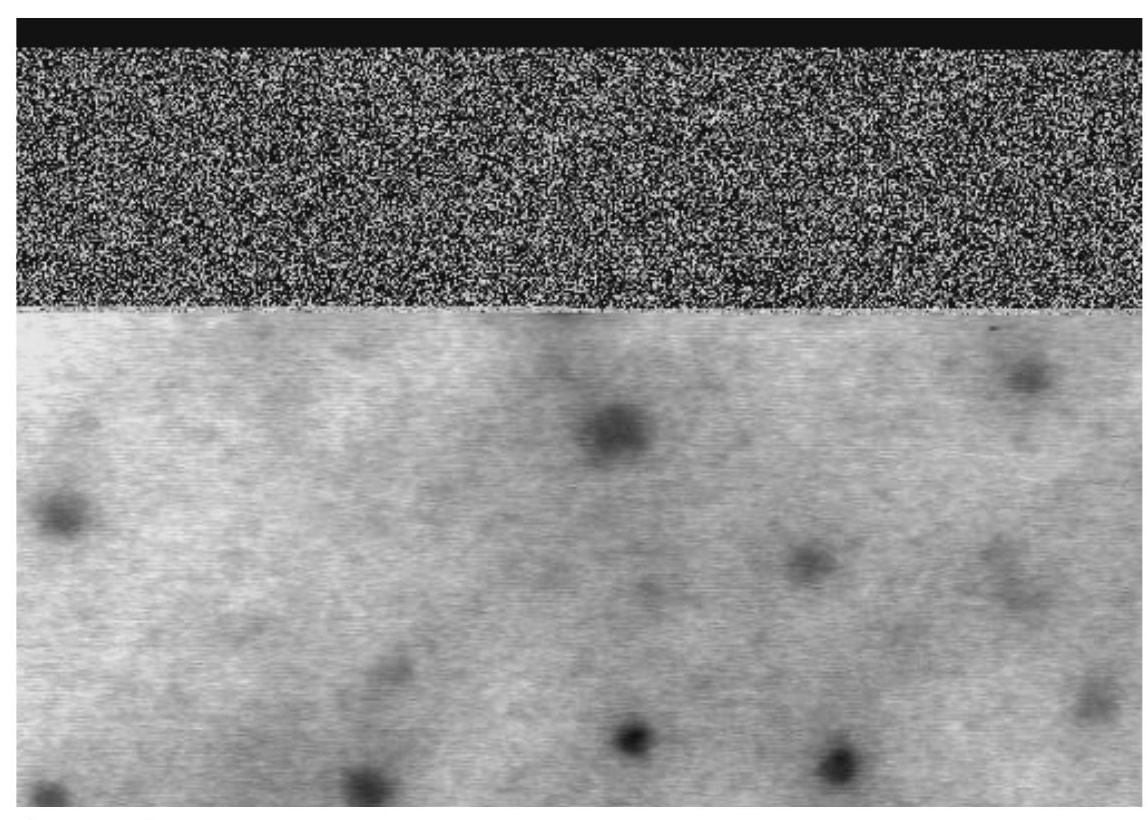

$200 \mathrm{~nm}$

Fig. 2. TEM picture of the micellar aggregates formed by the P2VP(26.4)-b-PEO(5.9) copolymer in water at $\mathrm{pH} 4.82$

Polydisperse spherical micelles are also observed for the P2VP(26.4)-b-PEO(5.9) sample at pH 4.82 (Fig. 2), although their characteristic size is much larger than the 
It must be pointed out that the characteristic structures shown in Figs. 3 and 4 coexist with smaller dense spherical micelles (see, e.g., Fig. 4) which could contribute very significantly to the scattering signal and account for a smaller $R_{\mathrm{h}}$ measured by DLS compared to the size estimated by TEM. All these morphologies have been previously observed for "crew-cut" micelles by Eisenberg et al. [6,13]

In order to gain deeper insight into the poorly defined micellar structure observed at $\mathrm{pH} 5.03$, additional DLS experiments have been carried out. In this respect, the angular (extrapolated to zero concentration) and concentration (extrapolated to zero angle) dependence of the apparent diffusion coefficient $\left(D_{\text {app }}\right)$ has been investigated for the micellar aggregates formed at $\mathrm{pH} 5.03$ (Fig. 5). The slope of the angular dependence of $D_{\text {app }}$ (Fig. 5a) is related to the shape of the diffusing species [14] and is found to be 0.022 consistent with the value predicted for a rod-like, worm-like or elliptical structure (0.03). Moreover, a deconvolution of the intensity autocorrelation function using the CONTIN routine shows a second slow relaxation mode characteristic of rod-like structures and resulting from the coupling of translational and rotational diffusion [15]. All these characteristic features are only observed at $\mathrm{pH} 5.03$ and clearly indicate the formation of rod-like micellar aggregates at this $\mathrm{pH}$. $D_{\text {app }}$ is found to be constant with concentration (Fig. 5b), in agreement with the formation of quasi-frozen micelles with extremely low critical micellar concentration. This conclusion is not surprizing because the P2VP core of these micellar aggregates is glassy at room temperature.
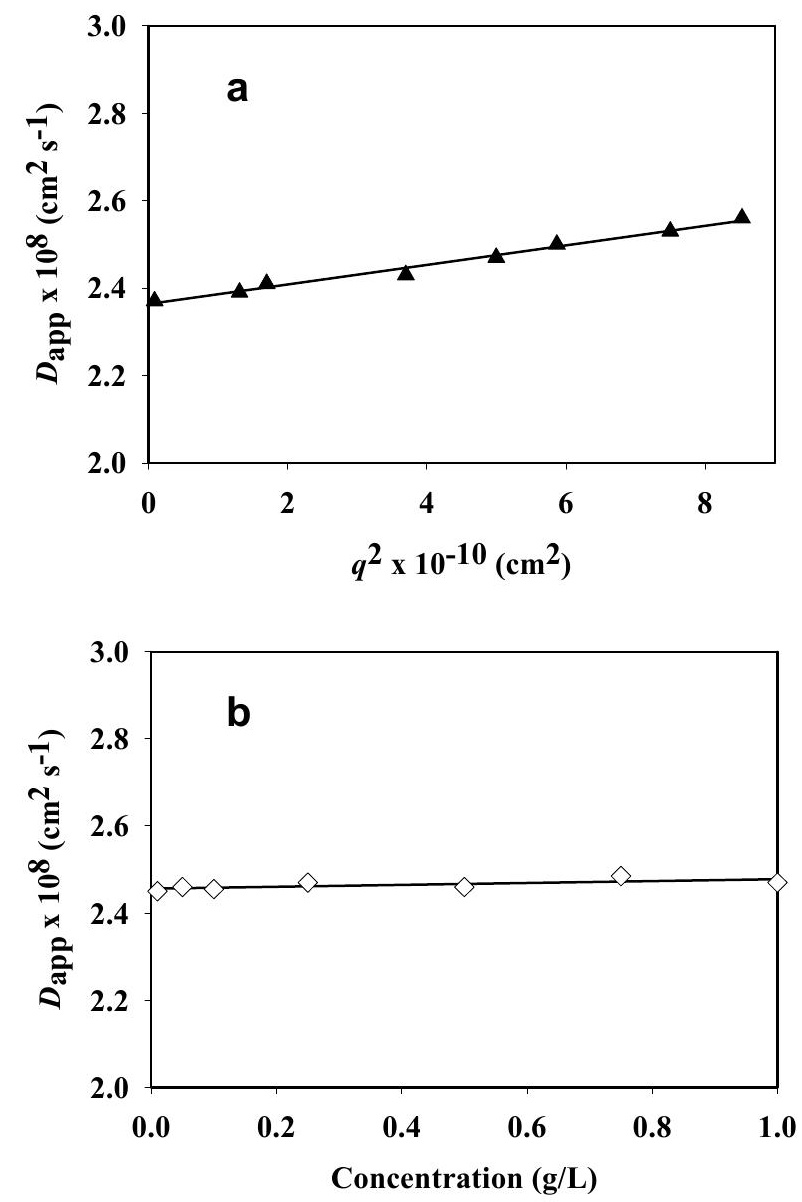

Fig. 5. Angular ( $a ; q$ is the scattering vector) and concentration (b) dependence of the apparent diffusion coefficient $\left(D_{\text {app }}\right)$ for the micellar aggregates formed at $\mathrm{pH} 5.03$ 
The sequence of structures shown by the P2VP(26.4)-b-PEO(5.9) sample when $\mathrm{pH}$ is increased from 4.9 to 5.5 , i.e., the spheres-to-rods-to-vesicles sequence is in qualitative agreement with Eisenberg et al. who reported the same sequence for "crew-cut" micelles formed by various copolymers $[6,13]$ in which the length of the hydrophobic block was constant and that of the hydrophilic block was decreased. According to these authors, the different micellar morphology results from the interplay of three factors, i.e., stretching of the core-forming blocks, surface tension between the micelle core and the solvent outside the core, and interaction between the coronal chains. In a homologous series of diblocks in water, the spheres-to-rodsto-vesicles sequence is consistent with the stretching of the core-forming blocks. In spherical micelles, the degree of stretching of the hydrophobic chains is proportional to the radius of the micellar core. The increase of this radius is however limited by the related loss in entropy, until the system becomes unstable and the morphology changes. Since the hydrophilic PEO block is the major constituent (83 mol-\%) of the P2VP(4.3)-b-PEO(9) sample (when all the 2VP units are deprotonated), star-like micelles are expectedly formed, that consist of a small hydrophobic P2VP core for which the problem of stretching of the constitutive chains is not critical.

The situation is quite different for the P2VP(26.4)-b-PEO(5.9) copolymer for which the hydrophilic PEO amounts to $35 \mathrm{~mol}-\%$ when all the 2VP units are deprotonated. This asymmetric composition can explain that star-like micelles are not stable in water. The mole fraction of all the hydrophilic moieties (EO and protonated 2VP units) has been calculated at $\mathrm{pH} 4.82,5.03$ and 5.19 and found to be $0.74,0.66$ and 0.60 , respectively. This calculation relies on the assumption that the mean $p K_{a}$ of the P2VP blocks is 5 , independently of the ionization degree, which is a rough approximation [8]. Therefore, at constant molecular weight, an increase in the mole fraction of the hydrophobic 2VP units from 0.26 to 0.40 triggers a spheres-to-rods-to-vesicles morphological transition. In the series of the polystyrene-block-poly(ethylene oxide) diblock copolymers studied by Eisenberg et al., the same sequence in morphology was observed in a much larger composition range, i.e., from 0.58 to 0.94 for the mole fraction of polystyrene [13a]. The two series of diblocks are thus not directly comparable. In the polystyrene-block-poly(ethylene oxide) series, the polystyrene length is constant and the PEO block length is increased. In the present contribution, one P2VP-b-PEO diblock forms a series when analyzed at different $\mathrm{pH}$ 's. Indeed, the PEO block length is constant and the number of hydrophobic 2VP units increases with increasing $\mathrm{pH}$. The basic difference is however that deprotonation of P2VP is a random process, such that the molecular structure of the "hydrophobic" block is complex and so should be its organization in the micellar core. In this respect, previous investigations on the ionization of homo-P2VP suggest that non-protonated 2VP units tend to self-assemble into hydrophobic domains [10].

It must be reminded that micellization only occurs in the presence of a phosphate buffer. This prerequisite is more likely related to the electrostatic repulsions between water-soluble charged 2VP units that segregate preferentially at the frontier with the aqueous phase. This situation is schematically depicted in Fig. 6, that shows a shell of water-soluble 2VP units around a micellar core of essentially uncharged hydrophobic 2VP units.

Addition of salt would be required to screen the strong electrostatic repulsions of the charged 2VP units, as it is sometimes the case for micellization of highly charged amphiphilic copolymers [16]. There is no reason for the phase separation between hydrophobic unprotonated and hydrophilic protonated P2VP to be sharp as ideally 
schematized in Fig. 6. This situation is consistent with poorly defined structures observed by TEM and coexistence of different morphologies at a given $\mathrm{pH}$.

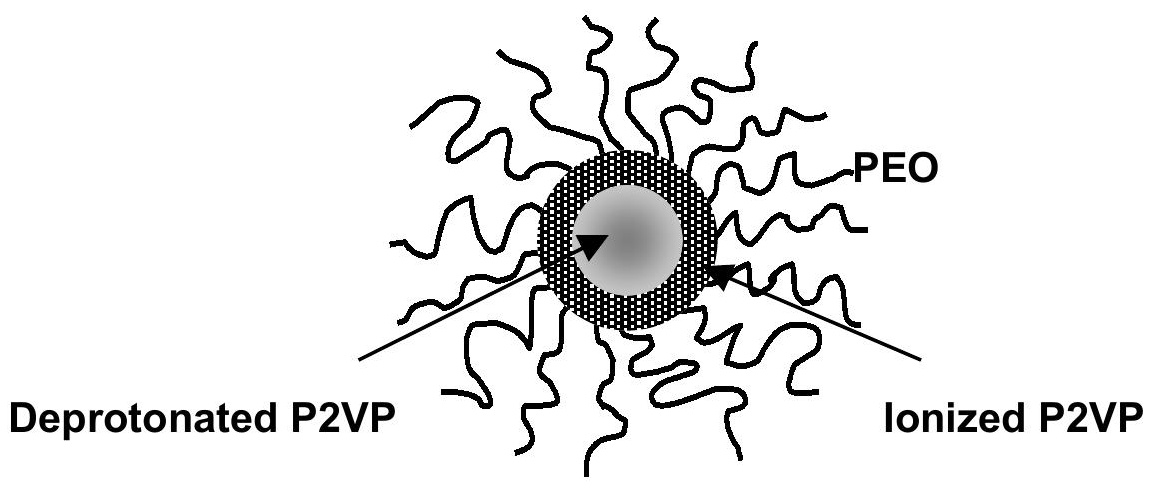

Fig. 6. Sketch for spherical micelles formed by the P2VP(26.4)-b-PEO(5.9) copolymer, in which part of the 2VP units is ionized

\section{Conclusions}

This contribution has shown that a spheres-to-rods-to-vesicles transition can be observed in the morphology of micelles formed by P2VP- $b$-PEO diblock copolymers that contain a major P2VP block, as a result of the careful control of the degree of ionization of P2VP. Charged 2-vinylpyridine units contribute to the hydrophilicity of the copolymer chains, which requires addition of screening salt for micellization to be observed.

The resulting micellar aggregates are less-defined than the ones previously reported for "crew-cut" micelles but, in the present investigation, all the morphologies could be reversibly obtained from a single copolymer by simply changing the $\mathrm{pH}$.

\section{Experimental part}

The P2VP(4.3)-b-PEO(9.0) and P2VP(26.4)-b-PEO(5.9) diblock copolymers, the numbers in parentheses being $\overline{M_{n}} \times 10^{-3}$, were synthesized by sequential living anionic polymerization with a polydispersity lower than 1.15 , as described elsewhere [11]. The copolymers where then directly dissolved in water, buffered at the requested $\mathrm{pH}$. A $0.1 \mathrm{~mol} / \mathrm{L}$ phosphate buffer was used and the copolymer concentration was $1 \mathrm{~g} / \mathrm{L}$.

Dynamic light scattering (DLS) measurements were performed on a Brookhaven Instruments Corp. BI-200 apparatus equipped with a BI-2030 digital correlator and an Ion Laser Technology argon laser with a wavelength of $488 \mathrm{~nm}$. The scattering angle used for the measurements was $90^{\circ}$. A refractive index matching bath of filtered water surrounded the scattering cell, and the temperature was controlled at $25^{\circ} \mathrm{C}$. The experimental intensity correlation function was measured and analysed by using a cumulant expansion, as described elsewhere [11]. The mean hydrodynamic radius $\left(R_{\mathrm{h}}\right)$, the angular dependence of the diffusion coefficient and the polydispersity of the micelles/aggregates were calculated accordingly.

Transmission electron microscopy (TEM) observations were carried out with a Philips CM 100 apparatus operating at $100 \mathrm{kV}$. TEM micrographs were directly recorded with a Gatan 673 CCD camera and data were transferred to a computer equipped with the 
Kontron KS 100 software. Samples were prepared by casting one drop of the aqueous micellar solution onto a Formvar-coated copper grid. Micelles were stained with $\mathrm{RuO}_{4}$ vapor for 30 min.

Acknowledgement: JFG is "Chargé de Recherches" by the Belgian National Foundation for Scientific Research (F.N.R.S.), and he thanks the European Science Foundation SUPERNET program. JFG, SM and RJ are very much indebted to the "Services Fédéraux des Affaires Scientifiques, Techniques et Culturelles" for financial support in the frame of the "Pôles d'attraction Interuniversitaires 4/11: Supramolecular Chemistry and Supramolecular Catalysis".

[1] Hamley, I. W.; "The Physics of Block Copolymers"; Oxford University Press, Oxford 1998, ch. 3.

[2] Creutz, S.; Jérôme, R.; Kaptijn, G. M. P.; van der Wert, A. W.; Akkerman, J. M.; Journal of Coating Technology 1998, 70, 41.

[3] (a) Yokoyama, M.; Inoue, S.; Kataoka, K.; Yui, N.; Sakurai, Y.; Makromol. Chem., Rapid Commun. 1987, 8, 431. (b) Yokoyama, M.; Okano, T.; Sakurai, Y.; Ekimoto, H.; Shibazaki, C.; Kataoka, K.; Cancer Res. 1991, 51, 3229.

[4] Discher, B. M.; Won, Y. Y.; Ege, D. S.; Lee, J. C. M.; Bates, F. S.; Discher, D. E.; Hammer, D. A.; Science 1999, 284, 1143.

[5] Gohy, J. F.; Creutz, S.; Garcia, M.; Mahltig, B.; Stamm, M.; Jérôme, R.; Macromolecules 2000, 33, 6378.

[6] Zhang, L.; Eisenberg, A.; Science 1995, 268, 1728.

[7] Martin, T. J.; Prochazka, K.; Munk, P.; Webber, S. E.; Macromolecules 1996, 29, 6071.

[8] Gohy, J. F.; Varshney, S. K.; Jérôme, R.; Macromolecules 2001, 34, 3361.

[9] Cölfen, H.; Macromol. Rapid Commun. 2001, 22, 219.

[10] (a) Puterman, M.; Koenig, J. L.; Lando, J. B.; J. Macromol. Sci. - Phys. 1979, B16(1), 89. (b) Puterman, M.; Garcia, E.; Lando, J. B.; J. Macromol. Sci. - Phys. 1979, B16(1), 117.

[11] Gohy, J. F.; Varshney, S. K.; Antoun, S.; Jérôme, R.; Macromolecules 2000, 33, 9298.

[12] Bronstein, L. M.; Sidorov, S. N.; Valetsky, P. M.; Hartmann, J.; Cölfen, J.; Antonietti, M.; Langmuir 1999, 15, 6256.

[13] (a) Yu, K.; Eisenberg, A.; Macromolecules 1996, 29, 6359. (b) Yu, K.; Zhang, L.; Eisenberg, A.; Langmuir 1996, 12, 5980. (c) Yu, K.; Eisenberg, A.; Macromolecules 1998, 31, 3509. (d) Yu, K.; Bartels, C.; Eisenberg, A.; Langmuir 1999, 15, 7157.

[14] Massey, J.; Power, K. N.; Manners, I.; Winnik, M. A.; J. Am. Chem. Soc. 1998, 120, 9533.

[15] Pecora, R. J.; J. Chem. Phys. 1968, 48, 4126.

[16] Baines, F. L.; Armes, S. P.; Billingham, N. C.; Tuzar, Z.; Macromolecules 1996, 29, 8151. 
Kontron KS 100 software. Samples were prepared by casting one drop of the aqueous micellar solution onto a Formvar-coated copper grid. Micelles were stained with $\mathrm{RuO}_{4}$ vapor for 30 min.

Acknowledgement: JFG is "Chargé de Recherches" by the Belgian National Foundation for Scientific Research (F.N.R.S.), and he thanks the European Science Foundation SUPERNET program. JFG, SM and RJ are very much indebted to the "Services Fédéraux des Affaires Scientifiques, Techniques et Culturelles" for financial support in the frame of the "Pôles d'attraction Interuniversitaires 4/11: Supramolecular Chemistry and Supramolecular Catalysis".

[1] Hamley, I. W.; "The Physics of Block Copolymers"; Oxford University Press, Oxford 1998, ch. 3.

[2] Creutz, S.; Jérôme, R.; Kaptijn, G. M. P.; van der Wert, A. W.; Akkerman, J. M.; Journal of Coating Technology 1998, 70, 41.

[3] (a) Yokoyama, M.; Inoue, S.; Kataoka, K.; Yui, N.; Sakurai, Y.; Makromol. Chem., Rapid Commun. 1987, 8, 431. (b) Yokoyama, M.; Okano, T.; Sakurai, Y.; Ekimoto, H.; Shibazaki, C.; Kataoka, K.; Cancer Res. 1991, 51, 3229.

[4] Discher, B. M.; Won, Y. Y.; Ege, D. S.; Lee, J. C. M.; Bates, F. S.; Discher, D. E.; Hammer, D. A.; Science 1999, 284, 1143.

[5] Gohy, J. F.; Creutz, S.; Garcia, M.; Mahltig, B.; Stamm, M.; Jérôme, R.; Macromolecules 2000, 33, 6378.

[6] Zhang, L.; Eisenberg, A.; Science 1995, 268, 1728.

[7] Martin, T. J.; Prochazka, K.; Munk, P.; Webber, S. E.; Macromolecules 1996, 29, 6071.

[8] Gohy, J. F.; Varshney, S. K.; Jérôme, R.; Macromolecules 2001, 34, 3361.

[9] Cölfen, H.; Macromol. Rapid Commun. 2001, 22, 219.

[10] (a) Puterman, M.; Koenig, J. L.; Lando, J. B.; J. Macromol. Sci. - Phys. 1979, B16(1), 89. (b) Puterman, M.; Garcia, E.; Lando, J. B.; J. Macromol. Sci. - Phys. 1979, B16(1), 117.

[11] Gohy, J. F.; Varshney, S. K.; Antoun, S.; Jérôme, R.; Macromolecules 2000, 33, 9298.

[12] Bronstein, L. M.; Sidorov, S. N.; Valetsky, P. M.; Hartmann, J.; Cölfen, J.; Antonietti, M.; Langmuir 1999, 15, 6256.

[13] (a) Yu, K.; Eisenberg, A.; Macromolecules 1996, 29, 6359. (b) Yu, K.; Zhang, L.; Eisenberg, A.; Langmuir 1996, 12, 5980. (c) Yu, K.; Eisenberg, A.; Macromolecules 1998, 31, 3509. (d) Yu, K.; Bartels, C.; Eisenberg, A.; Langmuir 1999, 15, 7157.

[14] Massey, J.; Power, K. N.; Manners, I.; Winnik, M. A.; J. Am. Chem. Soc. 1998, 120, 9533.

[15] Pecora, R. J.; J. Chem. Phys. 1968, 48, 4126.

[16] Baines, F. L.; Armes, S. P.; Billingham, N. C.; Tuzar, Z.; Macromolecules 1996, 29, 8151. 